\title{
EXTRATIVISMO, CULTIVO E PRIVATIZAÇÃO DO JABORANDI (PILOCARPUS MICROPHYLLUS STAPF EX HOLM.; RUTACEAE) NO MARANHÃO, BRASIL
}

\author{
Claudio Urbano B. Pinheiro ${ }^{1}$
}

Recebido em 06/07/2001. Aceito em 22/07/2001.

\begin{abstract}
RESUMO - (Extrativismo, cultivo e privatização do jaborandi (Pilocarpus microphyllus Stapf ex Holm.; Rutaceae) no Maranhão, Brasil). Um dos processos associados com a expansão de fronteiras em países em desenvolvimento consiste na privatização de recursos naturais, anteriormente de uso comum por populações tradicionais. Em geral, a privatização não só conduz à degradação dos recursos como também ao empobrecimento de comunidades locais dependentes desses recursos. Um exemplo desta transformação está no Estado do Maranhão, especialmente em uma região conhecida como "Pré-Amazônia", onde o jaborandi (Pilocarpus microphyllus Stapf ex Holm.; Rutaceae), um recurso natural que beneficiava milhares de pessoas da zona rural, foi gradativamente privatizado. A expansão do mercado de pilocarpina (um alcalóide de extenso uso em oftalmologia) nos últimos 30 anos, provocou crescimento descontrolado no extrativismo de folhas de jaborandi, conduzindo ao esgotamento e à ameaça de extinção das populações naturais deste recurso vegetal. Paralelamente, esta pressão sobre as populações naturais acelerou o processo de domesticação desta espécie e a sua privatização pela indústria farmacêutica. Este estudo teve como objetivo principal avaliar a exploração e cultivo do jaborandi no Maranhão, buscando o esclarecimento sobre os benefícios e malefícios econômicos, sociais e ambientais decorrentes da sua exploração. Os resultados obtidos mostram que, historicamente, não houve nenhum benefício significativo compartilhado entre a indústria farmacêutica e as populações envolvidas no extrativismo do jaborandi no Maranhão.
\end{abstract}

Palavras-chave - Pilocarpus microphyllus, Extrativismo, Cultivo, Privatização, Maranhão

\begin{abstract}
Extractivism, cultivation and privatization of jaborandi (Pilocarpus microphyllus Stapf ex Holm.; Rutaceae) in Maranhão, Brazil). A process associated with frontier expansion in developing countries is the privatization of natural resources formerly held in common by traditional populations. An example of this transformation comes from Maranhão State, in a region of mid-northern Brazil known as "Pre-Amazônia" (PreAmazon), where jaborandi (Pilocarpus microphyllus Stapf ex Holm.; Rutaceae), a plant resource that used to benefit thousands of rural poor, is being increasingly privatized. This process began with the expansion of the pilocarpine market in the world, provoking uncontrolled growth in extraction of native plants and resource depletion. This paper reports the latest developments regarding the exploitation of P. microphyllus in Maranhão, describing the current system of production, from leaf collection to industrialization. It also discusses social, economic and environmental benefits/damages to the people who exploited jaborandi and to the natural populations of this plant species. The results showed that, historically, there were no significant benefits shared between the pharmaceutical industry and people in the past.
\end{abstract}

Key words - Pilocarpus microphyllus, Extractivism, Cultivation, Privatization, Maranhão

\footnotetext{
${ }^{1}$ Universidade Federal do Maranhão, Departamento Oceanografia e Limnologia, Avenida dos Portugueses s/nCampus do Bacanga. CEP 65080-040 São Luís - Maranhão - Brasil.

Fone: (098) 217-8547 / Fax: (098) 217-8546 e-mail: cpinheiro@elo.com.br
} 


\section{Introdução}

Uma das drogas mais importantes em oftalmologia é derivada das folhas de um arbusto da família dos citrus, colhidas por índios e camponeses nas florestas do Brasil. Jaborandi (Pilocarpus spp.; Rutaceae - Pilocarpinae) é um arbusto ou arvoreta de 3-7,5 m de altura, de grande ocorrência no Brasil, desde o norte do Pará ao Rio Grande do Sul (Joseph 1967). Plantas de várias famílias já foram classificadas sob o nome jaborandi, principalmente aquelas que apresentam propriedades fisiológicas semelhantes, como algumas espécies de Piper (Piperaceae), Verbena (Verbenaceae) e Herpestis (=Bacopa, Scrophulariaceae), e até mesmo algumas outras espécies Rutaceae como os gêneros Esenbeckia, Zanthoxylum e Monnieria (Joseph 1967; Holmstedt et al. 1979). Contudo, o verdadeiro jaborandi pertence ao gênero Pilocarpus, da família Rutaceae, com 18 espécies descritas para o Brasil, de acordo com Joseph (1967) ou somente 10 espécies, de acordo com Kaastra (1982).

O jaborandi tem sido, durante as últimas três décadas, uma das espécies comerciais mais importantes da flora nativa brasileira. É a única fonte natural da droga pilocarpina, um alcalóide usado na oftalmologia para contração da pupila, importante em certos procedimentos cirúrgicos ópticos. Também é usada no tratamento de certos tipos de glaucoma. A pilocarpina é também um poderoso estimulante da salivação e da transpiração (Merck 1989); em 1994 foi aprovada pela Food and Drug Administration (FDA) norte-americana para o tratamento de xerostomia de pós-irradiação (boca seca) em pacientes com câncer de cabeça e de pescoço (Valdez et al. 1993; Joensuu et al. 1993; Rieke et al. 1995; Wynn 1996).

O jaborandi foi primeiramente apresentado à medicina ocidental em 1873, quando o português Symphrônio Coutinho levou a folha prova para a Europa. Seu valor em oftalmologia foi, de fato uma descoberta secundária. $\mathrm{O}$ que primeiro despertou o interesse dos médicos franceses foi o seu uso entre os índios brasileiros, para causar suor profuso e salivação. Ironicamente, este efeito no sistema nervoso que foi abandonado por tão longo tempo, retornou recentemente ao uso clínico.

$\mathrm{O}$ grande centro de produção de folhas de jaborandi no Brasil é o Estado do Maranhão, que produz cerca de $95 \%$ de toda a produção nacional (IBGE 1975-1998). Três espécies são descritas como de ocorrência no Maranhão: $P i$ locarpus jaborandi Holmes, P. trachyllophus Holmes e P. microphyllus Stapf ex Holmes (Joseph 1967), embora só esta última seja citada por Kaastra (1982), como de ocorrência naquele Estado. Pilocarpus microphyllus é considerado o "jaborandi legítimo" (Corrêa 1969) ou "jaborandi do Maranhão" por causa de sua ocorrência mais intensa no Maranhão e é reputado (e provado em laboratório) ter o mais alto nível de alcalóide em suas folhas. Outras espécies também contêm pilocarpina em concentrações variadas (uma média de 0,5\%), mais a mesma quantidade de outros alcalóides (isopilocarpina, jaborina, jaboridina e pilocarpidina); as folhas de P. microphyllus também contêm $0,24 \%$ a $0,38 \%$ de um óleo essencial com um cheiro de bálsamo (Merck 1983).

A companhia farmacêutica alemã, Merck, tem detido um monopólio de três décadas na compra de folhas de jaborandi e produção de pilocarpina no Brasil, mais particularmente no Maranhão. Desde o início (em 1969), estava claro para a Merck que o estoque natural de folhas de Pilocarpus poderia acabar. Eles concluíram que a única solução seria a domesticação do recurso natural, um processo que começou com os passos básicos de prospecção, coleta e avaliação de material silvestre de jaborandi. Este trabalho avançou com a aquisição, em 1989, de 2.250 ha de uma propriedade rural no município de Barra do Corda, na região da Pré-Amazônia Maranhense (SUDEMA 1970; Fig. 1). Lá, 
foram realizadas experiências para a seleção das melhores plantas que forneceriam o material genético para implementação do próximo e comercialmente mais importante passo: o cultivo de jaborandi.

Este estudo teve como objetivo principal a compreensão e descrição dos sistemas de produção praticados por caboclos (i.e., os habitantes tradicionais das regiões de estudo) e grupos indígenas. Adicionalmente, procurou-se localizar as principais áreas de produção nas diferentes regiões ecológicas do Maranhão, com a coleta de dados de produção dos últimos 23 anos para cada município no Estado, para elucidação dos diversos aspectos relacionados ao sistema de produção, áreas de produção e quantidades produzidas.

\section{Material e métodos}

O sistema atual de produção no Maranhão, das fases iniciais de coleta de folhas até a industrialização, foi avaliado. O trabalho de campo resultou no mapeamento botânico de P. microphyllus, realizado segundo a constatação da ocorrência das populações naturais e/ou em informação da sua ocorrência no passado. Coletou-se informações sobre o habitat, crescimento, produção de folhas e reprodução das plantas de ocorrência natural. Não foram efetuadas coletas botânicas por tratar-se de uma única espécie (P. microphyllus) já coletada e depositada em herbário nacional (UFMA) e estrangeiro (New York Botanical Garden). Seis viagens foram realizadas para as áreas de produção, nas diferentes regiões ecológicas de ocorrência do jaborandi, em um período de dois anos: três viagens para a Pré-Amazônia, duas para o Cerrado, e uma para o Litoral (Fig. 1). O número de viagens para cada região definiu-se a partir do volume de trabalho necessário em cada uma delas e pela necessidade de viagens adicionais. Foram ouvidos 36 informantes, entre coletores, intermediários e pessoal da fazenda da compa- nhia farmacêutica (agrônomos e operários). Foram realizadas entrevistas informais e semi-estruturadas. Dados secundários de produção também foram coletados. Os dados obtidos para um período de 23 anos mostraram a dinâmica da exploração do jaborandi no Maranhão e tornaram possível o entendimento da economia do jaborandi neste Estado. As informações sobre a plantação foram obtidas em visita à Fazenda Chapada, com os agrônomos da companhia. Ao final, compararam-se as informações obtidas durante o trabalho de campo e as entrevistas com os dados obtidos de fontes secundárias.

\section{Resultados e discussão}

O Sistema Tradicional - Não há diferenças significativas entre os sistemas de extração praticados nas diferentes regiões do Estado. O sistema extrativista pode ser, em geral, descrito como segue. Durante a estação seca (julho - dezembro), os coletores de folhas de jaborandi entram nas matas para a coleta de folhas das plantas de jaborandi. Eles afirmam que as plantas toleram este procedimento e que novas folhas rebrotam depois da colheita, quando do início da estação chuvosa. Entretanto, a coleta excessiva e freqüente de folhas da mesma planta ou grupo de plantas não permite o desenvolvimento completo das folhas novas, causando uma elevada percentagem de mortalidade de plantas, redução considerável na altura e vigor das plantas, bem como a redução no tamanho das novas folhas rebrotadas (Pinheiro 1997).

Uma variação nesse sistema é praticada pela Merck. O sistema da companhia está baseado em uma organização de fornecedores de folhas que recrutam homens para entrar nas florestas e coletar as folhas. Eles usam o mesmo método de retirada manual de folhas, mas afirmam que só estimulam a coleta durante a estação seca, por uma série de razões: 1) a penetração na floresta e secagem das folhas durante a estação chuvosa são mais difíceis (secagem mais lenta 


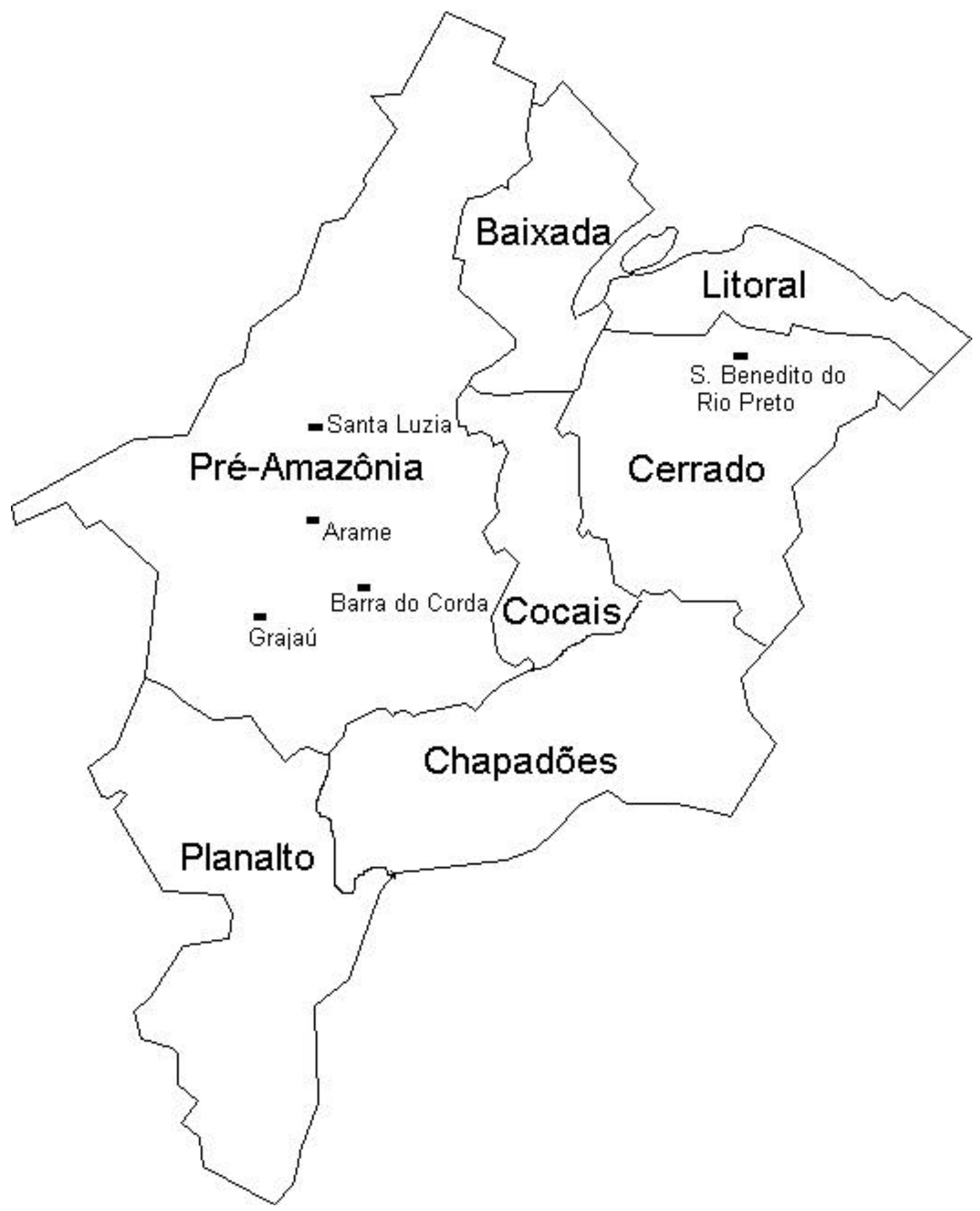

Figura. 1. Regiões Ecológicas do Maranhão - Localização das Áreas de Extrativismo e a Plantação da Companhia Farmacêutica (Barra do Corda). 
resulta em perda de conteúdo do alcalóide e custos operacionais mais altos; 2) na estação chuvosa, a mão-de-obra é mais difícil de recrutar (os coletores estão envolvidos em atividades agrícolas); e 3) para fins de conservação, a compra de folhas, na estação chuvosa, é suspensa para permitir que as plantas tenham um período de recuperação.

Na estação seca, o conteúdo de umidade nas folhas é de, aproximadamente, $40 \%$ e as folhas devem ser secadas até $10-12 \%$, pois um alto conteúdo de umidade pode causar fermentação e combustão espontânea durante o armazenamento (Frazão \& Pereira 1979). A maioria dos coletores simplesmente secam as folhas ao ar livre, espalhando-as no chão, sobre plástico. A secagem é feita sob luz solar direta, sendo necessários cerca de dois dias ou até que elas cheguem a uma cor creme-esverdeada. À noite, as folhas são mantidas em sacos impermeáveis, sob abrigos para evitar a reabsorção de umidade do ar.

Após a secagem, as folhas são limpas manualmente, removendo-se galhos e outro materiais; depois da limpeza, as folhas são colocadas em sacos de juta ou polipropileno. Os sacos são então transportados, normalmente por animais, das áreas de coleta para pontos de comércio fora das matas. As folhas de jaborandi são compradas com base no seu peso seco; em 1997, os compradores estavam pagando entre US\$2,50 - 4,00 por quilo de folhas, dependendo da classificação das mesmas. Na etapa final, as folhas são transportadas em caminhões para a fábrica da Merck em Parnaíba, no Estado do Piauí.

Jaborandi em Cultivo - Em 1989/90, a Merck adquiriu uma área de 2.250 ha, conhecida como Fazenda Chapada, no município de Barra do Corda (Fig. 1), a 350 km de São Luis.

Nessa época, a companhia farmacêutica plantou 3.000.000 de mudas de jaborandi, em cerca de 300 ha, sendo que a primeira colheita de folhas foi realizada em 1993. Desde então, muitas melhorias e investimentos foram efetua- dos para maximizar a produção e a produtividade das plantações da fazenda.

A área plantada com jaborandi na Fazenda Chapada, praticamente dobrou nos últimos cinco anos, mostrando os esforços e investimentos feitos pela companhia farmacêutica, baseados na certeza do crescimento e estabilidade do mercado de pilocarpina. O número de plantas de jaborandi na fazenda é cinco vezes maior que cinco anos atrás, em função dos avanços na tecnologia de produção da companhia. As áreas são plantadas atualmente, com maior adensamento (48.000 plantas/ha, em contraste com 23.000/ ha, no princípio do projeto), com fertilização química mais eficaz e sistematicamente realizada por meio de irrigação, além do uso do controle biológico de pragas (com emas e galinhas d'angola) e de nematóides (por inoculação da bactéria Pasteuria penetrans).

Atualmente, há cerca de 15 milhões de plantas de jaborandi (plantadas em aproximadamente $500 \mathrm{ha}$ ), que produzem em torno de $10.000 \mathrm{~kg}$ de folhas por ano. A produtividade alcança 1.800 $\mathrm{kg} / \mathrm{ha} /$ colheita, com 5-8 colheitas anuais. Esta produtividade é cinco vezes maior do que a obtida na primeira colheita de folhas, em 1993. Com um conteúdo médio de $1 \%$ de pilocarpina nas folhas de jaborandi, a produção anual da fazenda alcançou em 1998 cerca de $1.000 \mathrm{~kg}$, segundo informação dos agrônomos da companhia. Dados publicados por um jornal local (Estado do Maranhão, 11 de julho de 1999), entretanto, mostraram que a produção total de pilocarpina da Vegetex (a subsidiária da Merck, sediada em Parnaíba, PI) chegou a alcançar até 9 toneladas anualmente, avaliadas em US\$ 15 milhões no mercado mundial, segundo o jornal.

Classificação Comercial da Folha - Com a finalidade de padronizar os preços das folhas de jaborandi, coletadas no Maranhão, a companhia farmacêutica estabeleceu quatro classes de folhas (A, B, C e D). A, é o tipo de maior valor e deve apresentar uma série de características desejáveis, as quais incluem: cor creme-esverde- 
ada, indicando um nível satisfatório de desidratação; $10-12 \%$ de umidade; tamanho grande; e, alto nível de pilocarpina (as folhas de P. microphyllus são mais valorizadas pelo alto conteúdo do alcalóide).

$\mathrm{O}$ estabelecimento e uso desses critérios foram o resultado da experiência e do conhecimento acumulados com o passar do tempo pela companhia farmacêutica. Atualmente, a Merck divide as regiões de produção de acordo com estas quatro classes de folhas e estabelece o preço pago pelas folhas com base na origem das mesmas. A maioria das áreas de produção, na Pré-Amazônia, produzem o tipo A ou B de folhas, em contraste com outras áreas, classificadas principalmente como tipos $\mathrm{C}$ e $\mathrm{D}$ (por exemplo, no Litoral e na maioria das áreas da região do Cerrado) (Fig.1). As regiões do Cerrado e parte da região do Litoral são, provavelmente, as áreas mais antigas de exploração do jaborandi. Isto é confirmado pelos coletores e compradores de folhas em atuação nessas áreas.

Como consequiência do longo período de extrativismo intensivo e da super-exploração, as seguintes situações podem ser observadas, segundo os dados da produção extrativa vegetal do IBGE (1975-1998):

1) O volume de folhas produzidas nos dias atuais (menos de 500 toneladas) é significativamente menor do que aquele registrado no período 1975-1985 (cerca de 2.000 toneladas); uma redução na ordem de mais de $75 \%$ (Fig. 2).

2) nas áreas mais antigas de exploração, as folhas de jaborandi recebem uma classificação de $\mathrm{C}$ ou $\mathrm{D}$; estas áreas, contudo, já produziram pelo menos o tipo B. A classificação atual reflete a intensidade e a antiguidade da exploração do recurso nestas áreas. O longo período e a intensidade da exploração nestas áreas causaram a redução tanto no tamanho das folhas (produzidas agora por plantas de menor porte) como no nível de alcalóide.

Ao nível das regiões ecológicas algumas mudanças são visíveis em termos de produção.
As principais áreas da região de Cerrado produziram cerca de 200 t/ano de folhas nos anos 70; hoje, esta produção é de cerca de 30 t/ano. Na região da Pré-Amazônia, a redução da produção tem ocorrido na mesma magnitude; nos anos 70 a produção regional ficava em torno de 1300-1500 t/ano; hoje, é de, aproximadamente, 200 t/ano.

Ao nível de municípios, as mudanças foram até mesmo mais dramáticas: a intensidade de coleta de folhas provocou o desaparecimento total do jaborandi em algumas áreas. A coleta de folhas de jaborandi em muitas áreas do Estado tornou-se apenas uma lembrança nas mentes das pessoas mais velhas.

Os municípios mais importantes em produção de folhas de jaborandi no Maranhão, segundo as regiões ecológicas do Estado, são:

Na região pré-amazônica (Fig. 1), os municípios de Santa Luzia (média de 600 toneladas anuais de folhas na década de 70), Barra do Corda (700 toneladas/ano nos anos 70) e Grajaú (300-500 toneladas anuais nos anos 70) têm sido, historicamente, não apenas os principais produtores de folhas, mas também os maiores produtores de folhas de maior valor (tipos A e B). Estes três municípios, mais o município de Arame (criado da divisão do território de Grajaú) foram os únicos na Pré-Amazônia a manter a produção em torno de 100 t/ano, cada, até 1996. Atualmente, apenas o município de Arame mantém a produção acima de 100 t/ano.

Na região do Cerrado, o município de São Benedito do Rio Preto (Fig.1) apareceu como principal produtor de folhas. Entretanto, a produção caiu de 200 t/ano em média, nos anos 70, para 30 t/ano até 1995; em 1996 não houve produção registrada, mas em 1997 e 1998, a produção ficou em torno de 60 toneladas. Nos últimos 10 anos, as folhas colhidas nesta região têm recebido classificação $\mathrm{C}$ ou $\mathrm{D}$. Os coletores de folha afirmaram que, nos idos dos anos 70 esta classificação era B. A coleta intensiva foi apontada como responsável pela diminuição no vo- 


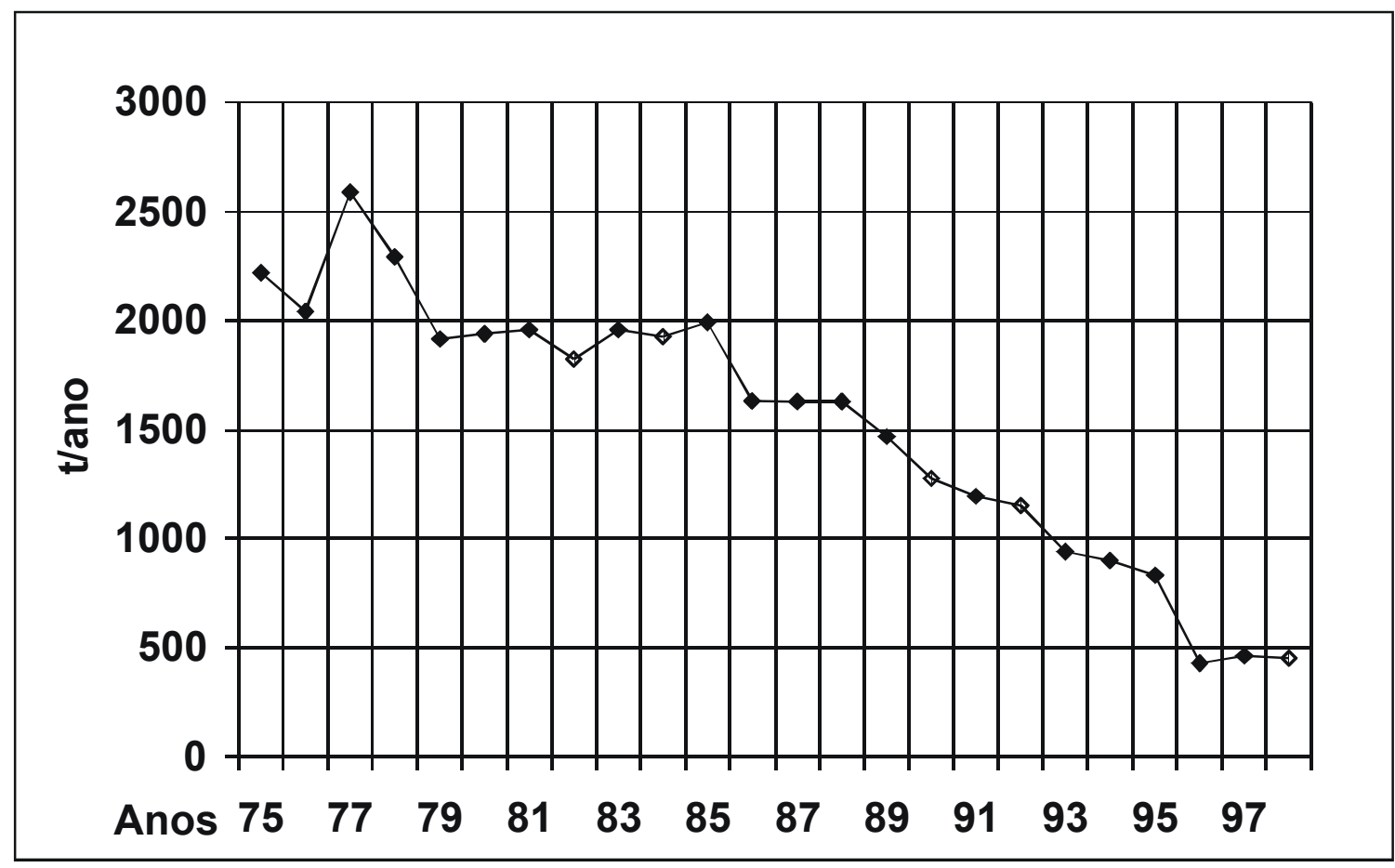

Figura 2. Produção Extrativa de Folhas de Jaborandi (t/ano) no Maranhão (IBGE: 1975-1998).

lume de produção e na qualidade das folhas produzidas na região.

O município de Barreirinhas, no Litoral Norte do Estado, apareceu nos dados de produção extrativa vegetal do IBGE, entre os anos de 1995 e 1998, como detentor de produção de folhas de jaborandi em patamares que variaram de 690 a 915 t/ano. Entretanto, o levantamento na área determinou que estes dados constituíram erros grosseiros do IBGE, pois a produção na área não passou, em nenhum dos anos nesse período, de uma tonelada.

Nos últimos anos (1998-2000), o mercado da pilocarpina também sentiu os problemas econômicos em várias partes do mundo. Tais problemas têm limitado o mercado e, como consequiência, a produção ficou estagnada. Os níveis de produção de pilocarpina natural também foram afetados pela produção da pilocarpina sintética a qual, até há pouco tempo, tinha utilização muito limitada, pelos efeitos colaterais indesejáveis apresentados no seu uso oftalmoló- gico. Esta situação, entretanto, mudou nos últimos dois anos e a pilocarpina sintética começou a ganhar espaço no mercado, que hoje se consolida.

Os investimentos incorporados e as melhorias tecnológicas promovidas pela indústria, para aumentar a produtividade e a produção de folhas, indicam que a pilocarpina continuou sendo um bom negócio por um longo período de tempo - pelo menos para a companhia farmacêutica. Para as populações locais, a coleta de folhas é, principalmente, uma substituição lucrativa para outras atividades diárias mais regulares, como a agricultura e outras formas de extrativismo. É levada a cabo por estas pessoas pelo fato de ser muito lucrativa, mas não é, em qualquer sentido, culturalmente incorporada ao seu modo de vida ou percebida como atividade econômica principal. Contudo, em algumas áreas tem sido observado que, por ter estado presente nas vidas destas comunidades por tão longo tempo, a atividade já apresenta um certo valor soci- 
al e relativa acomodação pela sociedade e sua cultura.

A pilocarpina foi usada originalmente por grupos indígenas como estimulante da salivação e da transpiração e, para estes fins a coleta de folhas era esporádica, sem fins lucrativos e não-destrutiva. Com o estabelecimento da companhia farmacêutica no Maranhão, há aproximadamente 30 anos, o processo induzido de exploração não-sustentável de jaborandi foi iniciado. Neste contexto, grupos indígenas e populações locais, em muitas áreas do Estado foram estimuladas (pelos preços pagos) à procura de plantas de jaborandi e, como resultado iniciou-se um processo consistente de degradação das populações naturais desta espécie.

A geração de tecnologia, para permitir o cultivo de jaborandi em pequenas áreas, poderia ter sido uma estratégia a ser usada para reduzir a pressão sobre as populações naturais. Isto também poderia ter contribuído para criar o conhecimento e as condições necessárias para pessoas locais mudarem de coletores para produtores de matéria-prima para a indústria, de um modo mais organizado e sustentável. As dificuldades para definir e implementar um sistema de produção nestes moldes são muitas, por exigir a acomodação de interesses de pequenos produtores e indústria. Duas estratégias diferentes foram consideradas e discutidas com pessoas envolvidas no extrativismo e no cultivo, como segue:

1) Plantio de jaborandi no sub-bosque das florestas pré-amazônicas - como tentativa de reproduzir as condições naturais onde estas plantas crescem nesta região. Esta poderia ser uma alternativa para grupos indígenas cultivarem plantas de jaborandi dentro dos limites de suas reservas. Contudo, vários problemas técnicos tornam este sistema desinteressante para a indústria: a) o tempo de colheita fica muito longo (10 anos) comparado ao obtido na plantação, sob luz solar direta (3 anos); b) a irrigação não é viável; c) não há meios para controle dos níveis de pilocarpina nas folhas (como é feito na plantação industrial, com o uso de cromatografia, segundo informado pelos agrônomos da Merck).

2) Cultivo de jaborandi em pequenas áreas associadas ou não com cultivos de subsistência ao nível dos pequenos produtores, alguns problemas aparecem como limitantes do sucesso desta alternativa:

a) pragas: vários insetos foram identificados como pragas do jaborandi em cultivo e os danos causados por alguns deles podem ser muito severos, como nos casos de gafanhotos e bichospau. O controle destes insetos pode ser difícil para pequenos produtores porque produtos químicos não podem ser usados. A Merck introduziu emas e galinhas d'angola nas áreas de plantação mantendo os níveis de infestação destas pragas sob condições razoáveis de manejo.

b) nematóides: o jaborandi (natural e cultivado) é susceptível a nematóides; deste modo, produtos químicos nematicidas não podem ser usados, porque se acumulariam nas folhas. Técnicas de manejo biológico (capinas reduzidas, uso de plantas antagônicas) foram estudadas pela indústria com resultados pouco satisfatórios obtidos para o controle deste problema. A adubação das plantas via água de irrigação contribuiu para minimizar o problema; os melhores resultados, porém, foram alcançados com a inoculação das plantas com a bactéria Pasteuria penetrans, um método menos acessível a pequenos produtores.

c) O controle do nível foliar de pilocarpina (como é realizado hoje, com o uso de equipamento sofisticado) não poderia ser mantido nas condições da pequena agricultura;

d) A operacionalização e os custos envolvendo a irrigação também podem ser limitantes.

A companhia farmacêutica não se interessou por um sistema de produção que pudesse aumentar a produção de folhas. Para estimular um sistema de pequenas plantações, a companhia teria que reduzir suas áreas plantadas. Isso 
não pareceu ser interessante para a indústria, uma vez dominada a tecnologia para produzir folhas de jaborandi com níveis controlados de pilocarpina, como resultado do expressivo investimento realizado ao longo das últimas três décadas.

A relação entre a companhia farmacêutica e as comunidades que foram levadas à coleta de folhas de jaborandi não parece ter sido uma relação bilateral em termos de benefícios diretos. O benefício pecuniário resultante da coleta e venda de folhas foi temporário (enquanto duraram as populações naturais da planta). Não houveram melhorias sócio-econômicas relevantes e duradouras trazidas pela exploração para as comunidades envolvidas. Pelo contrário, populações inteiras de jaborandi foram reduzidas ou destruídas. Melhorias sociais diretas seriam esperadas na área de influência da fazenda da companhia farmacêutica, pelo menos com a geração de um número expressivo de empregos. A expectativa de emprego, porém, foi maior apenas no início da implantação da plantação, a qual tornou-se, mais tarde, quase que totalmente mecanizada, dispensando grande parte da necessidade da mão-de-obra local.

Os benefícios econômicos para o Estado do Maranhão também são reduzidos e limitados ao Imposto sobre Circulação de Mercadorias e Serviços (ICMS), já que o Imposto sobre Produto Industrializado (IPI), é recolhido pelo Estado do Piauí, onde as folhas são processadas e de onde a pilocarpina é exportada. Assim, nem o Maranhão nem a sua gente receberam os lucros e benefícios por ter, ao longo de quase trinta anos, populações inteiras deste importante recurso vegetal devastadas, chegando a ponto do jaborandi estar entre as espécies da flora brasileira em perigo de extinção (IBAMA 1992). A companhia farmacêutica parece ter monopolizado a exploração, os lucros e os benefícios que deveriam ter sido distribuídos mais uniformemente entre as pessoas e comu- nidades que estiveram envolvidas na exploração do jaborandi. Além disso, a companhia farmacêutica parece preparar-se hoje, para substituir a produção de pilocarpina natural pela sintética. Como conseqüência, certamente, restarão apenas os danos ecológicos irreversíveis com a destruição das populações naturais de Pilocarpus no Maranhão e o mau exemplo do uso exaustivo deste recurso vegetal, sua privatização e finalmente, seu abandono.

\section{Agradecimentos}

Agradeço ao Conselho Nacional de Desenvolvimento Científico e Tecnológico - CNPq pela concessão de uma bolsa de Produtividade em Pesquisa, durante a vigência da qual este trabalho foi realizado.

\section{Referências Bibliográficas}

Corrêa, M.P. 1969. Dicionário das plantas úteis do Brasil, Vol. 4, Ministério da Agricultura, Rio de Janeiro. pp. 360-369.

Frazão, J.M.F. \& Pereira, R. L. S. 1979. Diagnóstico preliminar do jaborandi no Maranhão. Secretaria de Recursos Naturais, Tecnologia e Meio Ambiente, ITEMA, São Luis, Maranhão.

Holmstedt, B., Wassén, S. H. \& Schultes, R. E.. 1979. Jaborandi: an interdisciplinary appraisal. Journal of Ethnopharmacology 1 (1) : 3-21.

IBAMA. 1992. (Instituto Brasileiro do Meio Ambiente e dos Recursos Naturais Renováveis). 1992.

Portaria No. 06N. Janeiro 15. Diário Oficial, Brasília. pp. 870-872.

IBGE. 1975-1998. Produção Extrativa Vegetal. 18 Vols. Fund. Brasileira de Geografia e Estatística.

Joensuu, H., Bostrom, P. \& Makkonen, T. 1993. Pilocarpine and carbacholine in treatment of radiationinduced xerostomia. Radiotherapy and Oncology 26 (1): 33-37.

Joseph, C. J. 1967. Revisão sistemática do gênero Pilocarpus (ssp. brasileiras). Mecânica Popular, Rio de Janeiro, Outubro, 1-9.

Kaastra, R. C. 1982. Pilocarpinae (Rutaceae). Flora Neotropica, Monograph \# 33. New York Botanical Garden; New York. 1982. 
MERCK. 1983. Index Merck. An encyclopedia of chemicals, drugs and biologicals. Merck \& Co. , Rahway, New Jersey.

MERCK. 1989. Index Merck. An encyclopedia of chemicals, drugs, and biologicals. Susan Budavari, Ed., Merck \& Co.; Rahway, New Jersey.

Pinheiro, C.U.B. 1997. Jaborandi (Pilocarpus spp., Rutaceae): A Wild Species and Its Rapid Transformation into a Crop. Journal for Economic Botany. 51 (1), 49 - 58.

Rieke, J. W., Hafermann, M. D., Johnson, J. T., Leveque, F. G., Iwamoto, R., Steiger, B. W., Muscoplat, C \& Gallagher, S. C. 1995. Oral pilocarpine for radiation- induced xerostomia: Integrated efficacy and safety results from two prospective randomized clinical trials. International Journal of Radiation Oncology Biology Physics 31 (3): 661- 669.

SUDEMA (Superintendência do Desenvolvimento do Maranhão). 1970. Novo Zoneamento do Estado do Maranhão. São Luis, MA.

Valdez, I. H., Wolff, A., Atkinson, J. C., Macynski, A. A. \& Fox, P. C.. 1993. Use of pilocarpine during head and neck radiation therapy to reduce xerostomia and salivary dysfunction. Cancer 71 (5) : 18481851.

Wynn, R. L. 1996. Oral pilocarpine (Salagen): A recently approved salivary stimulant. General Dentistry 44 (1): 29-30. 